\title{
Crenças de autoeficácia e carreira de estudantes de administração
}

Self-efficacy beliefs and business administration student's careers

\author{
PEREIRA, Ana Lucia \\ Pontifícia Universidade Católica de São Paulo \\ analuciapereira@ig.com.br
}

ZUPPANI, Tatiani dos Santos

Faculdade de Economia, Administração e Contabilidade da Universidade de São

Paulo

tszuppani@gmail.com

GONÇALVES, Marcelo Neves

Faculdade de Economia, Administração e Contabilidade da Universidade de São

Paulo

marcelonevez@usp.br

\section{RESUMO}

As crenças de autoeficácia podem influenciar a maneira como os estudantes encaram a graduação, comportam-se no decorrer dela e se lançam aos desafios do mercado de trabalho. Nesse contexto, essa pesquisa buscou identificar as variáveis preditoras das crenças de estudantes de Administração em relação ao curso superior, buscando contribuir com os estudos sobre as variáveis controláveis que impactam no aproveitamento acadêmico e na inserção profissional dos universitários. Para tanto, foi realizado um survey junto a 585 alunos de Administração. O questionário foi composto pelas variáveis independentes: gênero, faixa etária, turno de estudo e semestre; e teve como variáveis dependentes as crenças dos universitários sobre carreira e graduação. Através de análises descritivas e de regressão logística, verificou-se que o turno e o semestre apresentam impacto nas crenças de autoeficácia. Essas crenças são mais positivas para os alunos que estudam à noite e estão finalizando o curso.

Palavras-chave: Crenças de autoeficácia; Carreira; Estudantes de administração. 


\section{ABSTRACT}

The self-efficacy beliefs may influence how the students face their degree, how they behave during their studies and how they face the challenges of the market. In this context, this research searched to identify the predictor variables of the Administration students beliefs related to the higher education, seeking to contribute with the controllable variable studies that impact with the academic results and with the graduates market beginning. To accomplish that, a survey was conducted with 585 Business Administration students. This survey was composed by independent variables: gender, age group, study period and semester; and had as dependent variable graduate beliefs about degree and carrier. Using descriptive analyzes and logistic regression, it was verified that the period and semester show impact on the self-efficacy beliefs. These beliefs are more positive for night students and students that are finishing the course.

Key Words: Self efficacy beliefs; career; administration students.

\section{Introdução}

A graduação, sobretudo para estudantes oriundos de classes sociais menos privilegiadas, é o caminho buscado para a ascensão financeira e social. É fato que essa busca é influenciada por variáveis que não são controláveis pelo estudante, tais como: escassez ou excesso de profissionais qualificados na profissão escolhida, demanda do mercado de trabalho por profissionais com aquela formação, impactos macroambientais que afetam o mercado de trabalho e assim por diante.

Entretanto, é igualmente verdadeira a existência de outra categoria de variáveis, relacionadas às crenças de autoeficácia dos alunos em relação ao seu desempenho no curso e aos resultados obtidos nele. Essas crenças podem influenciar fortemente a maneira como os estudantes encaram a graduação, comportam-se no decorrer dela e se lançam aos desafios do mercado de trabalho.

As crenças de autoeficácia, que ocupam um papel de destaque na Teoria Social Cognitiva de Bandura (1992), consistem nas percepções das pessoas a respeito de suas próprias capacidades, sendo elemento fundamental na constituição da motivação humana e impactando nas realizações acadêmicas profissionais. Dessa forma, para o autor, quanto mais elevada for a crença de autoeficácia da pessoa, maior será a sua facilidade para obter êxito em circunstâncias que exigem 
esforço pessoal, a sua tolerância a frustrações e a sua persistência para contornar os desafios que surgirem no decorrer do percurso acadêmico.

Diante do exposto, este trabalho tem como objetivo identificar as variáveis preditoras das crenças de estudantes de Administração em relação ao curso superior, de modo a revelar indicadores que possam impactar na percepção de comportamentos e mudanças que interfiram no desenvolvimento de suas carreiras.

Pretende-se, dessa forma, contribuir com os estudos sobre as variáveis controláveis que impactam no aproveitamento acadêmico e na inserção profissional de estudantes universitários, eventualmente trazendo pistas para a elaboração de estratégias de intervenção junto aos estudantes em diversos estágios do curso.

\section{Crenças no contexto da teoria social cognitiva}

A teoria proposta por Albert Bandura foi inicialmente nomeada como Teoria da Aprendizagem Social. Entretanto, na medida em que os estudos do autor avançaram, seu conceito de aprendizagem foi ganhando um caráter mais abrangente, levando em consideração o papel ativo das pessoas. Nessa perspectiva mais ampla, a teoria foi rebatizada como Teoria Social Cognitiva.

Como fruto da abordagem behaviorista de Skinner, ela se concentra em comportamentos manifestos; mas diferente desta, a Teoria Social Cognitiva assume a existência de variáveis cognitivas mediadoras entre os estímulos do ambiente e as respostas emitidas (comportamentos manifestos) pelo indivíduo.

A Teoria Social Cognitiva enfatiza a importância dos processos cognitivos para a motivação, a emoção, as ações e os comportamentos dos seres humanos. Para consolidar essa perspectiva, Bandura promoveu investigações com alto grau de sofisticação e rigor experimental, por meio da observação do comportamento humano em laboratórios e em ambientes sociais (SCHULTZ; SCHULTZ, 2008).

Dentre as variáveis cognitivas estudadas por Bandura (1997) merecem destaque as crenças relacionadas com a autoeficácia ou capacidade percebida de lidar com situações específicas. De acordo com o estudioso, essas crenças são construídas a partir de experiências de sucesso ou fracasso, sendo que um histórico de fracassos sucessivos contribui para a queda da autoconfiança e, em consequência, reduz as crenças de autoeficácia do indivíduo. 
Dessa forma, as crenças de autoeficácia têm potencial de interferência direta nas atividades das pessoas, podendo impactar de maneira quantitativa e qualitativa nos seus esforços e na persistência dedicados às tarefas cotidianas, bem como nas reações emocionais diante de dificuldades e obstáculos.

A Figura 1 ilustra a relação dialética entre as crenças de autoeficácia, os pensamentos, as emoções e o comportamento dos indivíduos.

Figura 1: Relações dialéticas entre as crenças de autoeficácia

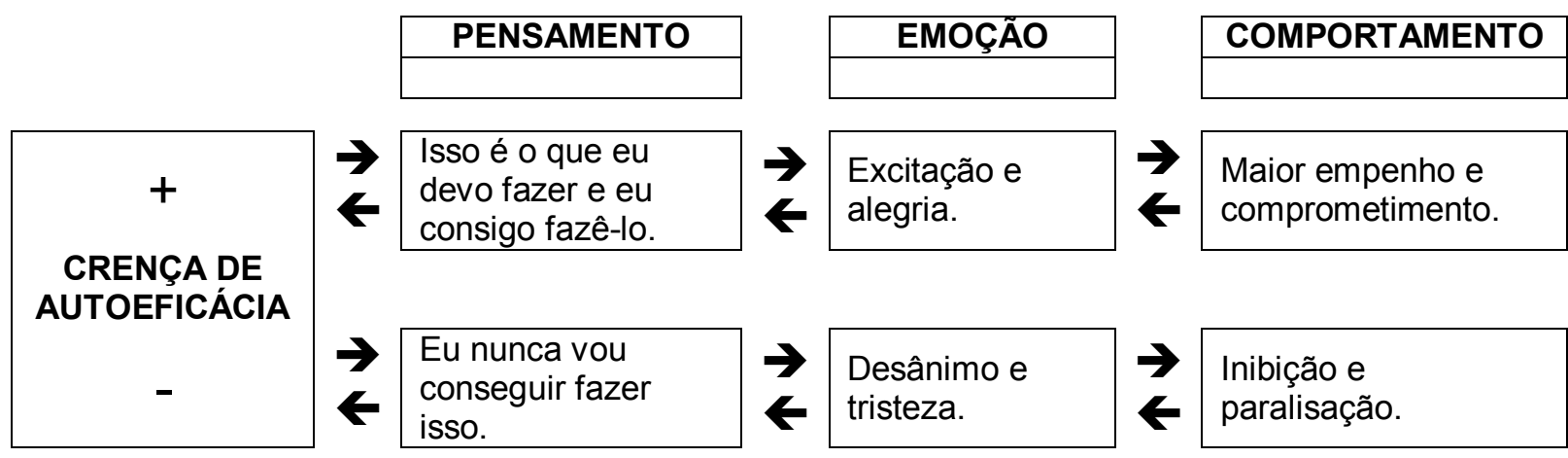

Fonte: Elaborado pelos autores.

De acordo com a Teoria Social Cognitiva, diante do mesmo ambiente potencial, os indivíduos com elevada crença de autoeficácia têm pensamentos e emoções que Ihes motivam a concentrar seus esforços nas oportunidades oferecidas, enquanto aqueles com reduzida crença de autoeficácia tendem a enfatizar as dificuldades e os riscos.

Sendo assim, quanto maior for a crença de autoeficácia percebida pelo indivíduo, menor será a sua dificuldade em situações estressantes ou que demandem esforço pessoal e persistência mais acentuados (BANDURA, 1987).

Bandura (1995, p. 1) também destaca a relação entre a autoeficácia e a percepção do indivíduo sobre a própria vida:

[...] as pessoas se esforçam para controlar os eventos que afetam a sua vida, pois quando adquirem influência sobre as variáveis que permitem certo controle, tornam-se mais capacitadas para concretizar os futuros que desejam e evitar os indesejados. [...] Esse controle sobre as influências ajuda a torná-las previsíveis, favorecendo uma preparação adequada; ao passo que sua ausência produz apreensão, apatia ou desespero.

Dessa forma, a baixa crença de autoeficácia pode levar à sensação de desamparo e à incapacidade de lidar com as adversidades da vida, levando o 
indivíduo a desistir precocemente de seus objetivos ou até mesmo de tentar dar os primeiros passos.

Para Schultz e Schultz (2008) as crenças de autoeficácia podem influenciar em aspectos como a quantidade de tempo dedicada na procura de um emprego e, posteriormente, nas ações necessárias para a obtenção de sucesso no futuro trabalho. Ou seja, pessoas com elevada crença de autoeficácia tenderiam a estabelecer objetivos acadêmicos e profissionais mais ousados e se empenhar para alcançá-los mais do que as com baixa crença de autoeficácia.

Os autores citam um estudo realizado por Stajkoviv e Luthans (1998) que consistiu em uma meta-análise de 114 estudos de pesquisa envolvendo aproximadamente 21.600 analisados, que indicou que quanto mais alto era o nível de autoeficácia das pessoas, melhor era o desempenho delas no trabalho.

Isso ocorre porque aqueles que apresentavam crença de autoeficácia elevada percebiam-se como capazes de lidar satisfatoriamente com os desafios e adversidades da vida acadêmica e profissional, tornando-se mais perseverantes em suas tarefas e em muitos casos apresentando desempenho de alto nível.

Diante do exposto, evidencia-se a importância de estudos que investiguem o impacto das crenças de autoeficácia de estudantes em suas carreiras.

\section{Procedimentos metodológicos}

Para alcançar o objetivo proposto neste artigo foi realizada uma pesquisa com abordagem quantitativa de corte transversal. Para tanto, foi realizado um survey, que é descrito por Hair et al. (2005) como um procedimento para coleta de dados primários a partir de uma grande quantidade de indivíduos.

Assim, foi aplicado um questionário estruturado de autopreenchimento a 585 alunos do curso presencial de Administração de uma Instituição de Ensino Superior (IES) privada localizada na cidade de São Paulo. Os alunos do curso de Administração da IES têm idade entre 23 e 27 anos, pertencem a famílias de classe social $\mathrm{C}$ e D e, em sua maioria, são oriundos do ensino público.

O questionário foi composto por dois conjuntos de variáveis. O primeiro conjunto foi composto pelas variáveis independentes do estudo, entre elas:

- Sexo: masculino ou feminino. 
- Faixa etária: entre 17 e 21 anos; entre 22 e 26 anos; entre 27 e 30 anos; ou acima de 30 anos.

- Turno de estudo: diurno ou noturno.

- Semestre: $1^{\circ}$ semestre; $4^{\circ}$ semestre; ou $8^{\circ}$ semestre, representando, respectivamente, o início, meio e final do curso.

O segundo conjunto de variáveis foi formado por 22 afirmações a respeito das atitudes dos estudantes em relação à sua carreira e graduação, formando-se, assim, as variáveis dependentes do estudo. Essas assertivas deveriam ser pontuadas pelos alunos em uma escala métrica com notas de 1 a 10 , na qual 1 significava "Discordo Totalmente" e 10 "Concordo Totalmente". Essa escala foi nomeada "Crenças sobre carreira e graduação acadêmica".

Os temas presentes nessa escala foram: a percepção do estudante quanto à empregabilidade após ter iniciado a graduação; a responsabilidade que os estudantes atribuíam a eles mesmos e ao curso na evolução de seu trabalho fora da universidade; as percepções de mudanças efetivas ocorridas em sua vida profissional e a metapercepção do estudante sobre o que a empresa enxerga do trabalho dele.

Buscou-se com essa escala identificar a crença dos respondentes sobre a relação entre o curso superior e a percepção de comportamentos e mudanças que interferem no desenvolvimento de suas carreiras. Para a análise dos dados foram utilizadas técnicas de análise descritiva e regressão logística, realizadas através do software estatístico SPSS 19.0 (Software Package Social Science).

\section{Resultados}

\section{Análise descritiva}

Para alcançar o objetivo proposto e realizar a técnica estatística de regressão logística utilizou-se a escala de Crenças sobre carreira e graduação acadêmica para classificar os respondentes em dois grupos diferentes segundo sua autoeficácia. Entendeu-se que uma pontuação alta revelava uma crença positiva dos estudantes quanto à sua autoeficácia em relação à sua carreira, enquanto uma pontuação baixa na escala mostrava uma crença negativa em relação à sua autoeficácia. 
Assim, foi criada uma média para cada respondente com base na variável métrica coletada, ou seja, foi retirada uma média das notas que cada respondente deu às vinte e duas assertivas da escala "Crenças sobre carreira e graduação acadêmica". Posteriormente, foi calculada uma média dessas médias individuais, chegando-se ao valor de 5,81.

Como essa variável seria a responsável pela criação dos diferentes grupos para o estudo, decidiu-se por retirar da amostra todos os respondentes com média um ponto acima ou abaixo da média geral $(5,81)$. Isso significa que todos os respondentes que obtiveram média entre 4,81 e 6,81 na escala de crenças foram excluídos da amostra, totalizando a exclusão de 238 respondentes, gerando dois grupos mais díspares. Dois indivíduos também foram excluídos da amostra por não terem respondido a questão de gênero.

Ao grupo com crenças positivas sobre sua autoeficácia foi dado o nome de "Alta autoeficácia" e ao grupo com crenças negativas foi atribuído o nome "Baixa autoeficácia". A distribuição dos indivíduos nos grupos é apresentada na Tabela 1.

Tabela 1 - Número de participantes dos grupos de baixa autoeficácia e alta autoeficácia

\begin{tabular}{c|c|c|c}
\hline Grupo & Faixa de Corte & $\mathbf{n}$ & $\%$ \\
\hline Baixa autoeficácia & Médias abaixo de 4,80 & 183 & 53,2 \\
Alta autoeficácia & Médias acima de 6,81 & 161 & 46,8 \\
\hline Total & - & 344 & 100 \\
\hline
\end{tabular}

Fonte: Elaborado pelos autores.

Dessa forma, obteve-se a variável categórica binária dependente que permitiu o uso da técnica de regressão logística. Ainda, dentro de uma perspectiva descritiva, foi realizada uma tabulação cruzada das variáveis independentes com os grupos criados. O resultado desse cruzamento é apresentado na Tabela 2.

Tabela 2: Cruzamento das variáveis independentes e os grupos de baixa e alta autoeficácia

\begin{tabular}{|c|c|c|c|c|}
\hline & Categorias & Baixa Autoeficácia & Alta Autoeficácia & Total \\
\hline \multirow{3}{*}{ Sexo } & Masculino & 67 & 50 & 117 \\
\hline & Feminino & 116 & 111 & 227 \\
\hline & Total & 183 & 161 & 344 \\
\hline Faixa Etária & 17 a 21 anos & 21 & 45 & 66 \\
\hline
\end{tabular}




\begin{tabular}{c|c|c|c|c} 
& 22 a 26 anos & 88 & 62 & 150 \\
& 27 a 30 anos & 48 & 26 & 74 \\
& Acima de 30 anos & 26 & 28 & 54 \\
& Total & 183 & 161 & 344 \\
\hline \multirow{4}{*}{ Turno } & Diurno & 154 & 38 & 192 \\
& Noturno & 29 & 123 & 152 \\
& Total & 183 & 161 & 344 \\
\hline \multirow{5}{*}{ Semestre } & $1^{\circ}$ semestre & 90 & 20 & 110 \\
& $4^{\circ}$ semestre & 83 & 54 & 137 \\
& $8^{\circ}$ semestre & 10 & 87 & 97 \\
& Total & 183 & 161 & 344 \\
\hline
\end{tabular}

Fonte: Elaborado pelos autores.

Em uma inspeção preliminar, parece haver homogeneidade no número de participantes dos grupos formados e as variáveis sexo e idade. No entanto, aparentemente, quando se compara a autoeficácia nos diferentes turnos e semestres verifica-se heterogeneidade. Os alunos que estudam pela manhã parecem ter crenças de sucesso menores e alunos que estudam à noite parecem acreditar mais em seu sucesso profissional. Com relação ao semestre, parece que com o transcorrer do curso universitário, mais crédulo de seu potencial o jovem fica.

\section{Regressão logística}

A regressão logística é uma forma especializada de regressão, que é formulada para prever e explicar uma variável dependente categórica binária (dois grupos). Ela representa uma relação multivariada, na qual os coeficientes indicam o impacto relativo de cada variável preditora (HAIR et al., 2005). Nessa técnica, a probabilidade de ocorrência de um evento pode ser estimada diretamente (MINUSSI; DAMACENA; NESS JR., 2002).

Antes da realização da técnica, foram testadas as premissas da regressão logística, que são normalidade, igualdade das variâncias e multicolinearidade. As duas primeiras premissas não foram atendidas, mas verificou-se uma baixa correlação entre as variáveis independentes. Apesar das premissas de normalidade e de homogeneidade das matrizes de variância e covariância não terem sido atendidas, a regressão logística é uma técnica robusta o suficiente para a quebra dessas suposições (HAIR et al., 2005). 
Por isso, procedeu-se com a análise. Para a realização da regressão logística, considerou-se:

- variável dependente: baixa autoeficácia e alta autoeficácia;

- variáveis independentes: sexo, faixa etária, turno de estudo e semestre; e

- procedimento de seleção das variáveis preditoras: método Stepwise.

Assim, o primeiro modelo considerou apenas a constante. No entanto, diante de um sig>0,05 e um valor baixo da estatística Wald, verificou-se que a constante não deve ser incluída sozinha no modelo. Diante dessa decisão, foi testado o modelo considerando as variáveis dependentes: sexo, gênero, turno e semestre; sendo este representado através de:

$\log$ it (autoeficácia $)=\alpha_{0}+\beta_{1}$ sexo $+\beta_{2}$ gênero $+\beta_{3}$ turno $+\beta_{4}$ semestre

Para verificar se as quatro variáveis independentes seriam adequadas ao modelo, utilizou-se o método stepwise, que utilizando a estatística Wald consumiu dois passos até obter o modelo final, com um coeficiente significante $(\operatorname{sig}<0,05)$ a cada passo. O modelo final, obtido no segundo passo, incluiu duas das quatro variáveis independentes estudadas.

O ajuste do modelo com duas variáveis também se mostrou melhor do que aquele incluindo todas. Verificou-se que com as variáveis turno e semestre, 0 modelo apresentou um ajustamento significativo $\left(X^{2} ; 4 \mathrm{gl}=48,553\right.$ e significância $=$ 0,000) e a medida de Nagelkerke foi de 0,694, quando o máximo possível a ser atingido é +1 . Tais resultados mostram bom poder de previsão do modelo.

As estimativas e significância das variáveis consideradas estatisticamente significativas para o modelo foram o turno e o semestre. Esses dados são detalhados na Tabela 3, seguidos pela taxa de acerto do modelo, apresentada na Tabela 4.

Tabela 3: Estimativas dos Coeficientes Beta e Testes de Significância

\begin{tabular}{c|c|c|c|c|c|c}
\hline Variáveis & $\boldsymbol{\beta}$ & $\begin{array}{c}\text { Erro } \\
\text { Padronizado }\end{array}$ & Wald & Gl & Sig. & Exp(B) \\
\hline Turno & $-3,873$ & 0,440 & 77,554 & 1 & 0,00 & 0,021 \\
Semestre & 2,454 & 0,292 & 70,387 & 1 & 0,00 & 11,63 \\
Constante & $-2,837$ & 0,497 & 32,634 & 1 & 0,00 & 0,059 \\
\hline
\end{tabular}

Fonte: Elaborado pelos autores. 
Tabela 4 - Tabela de estimativa de previsão de acertos

\begin{tabular}{|c|c|c|c|c|}
\hline & \multicolumn{4}{|c|}{ Previsto } \\
\hline & & $\begin{array}{c}\text { Baixa } \\
\text { autoeficácia }\end{array}$ & Alta autoeficácia & $\%$ \\
\hline \multirow[t]{2}{*}{ Observado } & Baixa autoeficácia & 166 & 17 & 90,7 \\
\hline & Alta autoeficácia & 24 & 137 & 85,1 \\
\hline & Total & & & 88,1 \\
\hline
\end{tabular}

Fonte: Elaborado pelos autores.

Esses dados em conjunto revelam que o modelo proposto apresenta uma boa qualidade de ajuste, pois com a inclusão das duas variáveis independentes no modelo, o número de previsões corretas foi de $88,1 \%$. Valor este superior aos acertos ao acaso que era de $53,2 \%$.

Assim, o modelo proposto é representado através de:

$$
\text { Log it (autoeficácia) }=-2,837-3,873^{*} \text { Turno }+2,454^{*} \text { Semestre }
$$

Tal função permite estabelecer a probabilidade $(P)$ da autoeficácia do aluno ser alta ou baixa em função do turno e semestre que o estudante se encontra. Isso permite trabalhar com a estimação de cenários futuros e desenvolver ações que possam ser mais positivas na tratativa de casos desfavoráveis.

\section{Considerações finais}

O presente estudo buscou testar as variáveis preditoras da crença de autoeficácia dos estudantes com relação ao curso superior, objetivando verificar se elas impactam na percepção de comportamentos e mudanças que interferem no desenvolvimento de suas carreiras. Foram estudadas como variáveis independentes o sexo, a faixa etária, o turno e o semestre de 585 estudantes do curso de Administração de uma IES da cidade de São Paulo e, como variável dependente, as crenças desses universitários sobre carreira e graduação acadêmica.

Para efetuar as análises foram utilizadas as técnicas estatísticas de análise descritiva e regressão logística e, pelos resultados, foi possível delinear um modelo de previsão da intensidade da autoeficácia desses estudantes com base no turno e semestre que eles se encontravam. 
A análise descritiva dos dados permitiu verificar que $80 \%(n=154)$ dos alunos que estudam no turno diurno e $24 \%(n=29)$ dos que estudam no noturno apresentaram crenças de baixa autoeficácia. Estudos futuros seriam necessários para investigar essa discrepância estatisticamente significativa. Entretanto, é importante considerar que os dados foram coletados em uma universidade privada voltada para o público $\mathrm{C}$, no qual muitos estudantes trabalham para pagar a mensalidade.

Por meio de observações informais, verifica-se que muitos dos alunos do diurno trabalham em atividades divergentes de sua área de estudo, sendo comum atuarem como garçons, porteiros, manicures, vigilantes ou no mercado informal. É prática habitual, quando conseguem colocações na área administrativa, solicitarem transferência para o período noturno, que, por sua vez, conta com maior número de alunos atuando como estagiários ou em cargos iniciais na administração. Dessa forma, sugere-se que pesquisas futuras verifiquem a correlação entre as crenças de autoeficácia e a posição do mercado de trabalho.

No que diz respeito ao semestre, constatou-se a crença de autoeficácia é maior entre os alunos do oitavo semestre $(90 \% ; n=87)$. Embora esse fosse um resultado esperado, tendo em vista a expectativa de que os último-anistas tenham a autopercepção de estarem mais preparados para o mercado de trabalho que os calouros ou estudantes que estão na metade do curso. Estudos futuros em IES com diferentes perfis de estudantes seriam recomendáveis.

Além de aprofundar os estudos com relação a essas duas variáveis (turno e semestre), também fica como sugestões para estudos futuros a inclusão de outras variáveis, como a alteração de turno durante o curso e o nível de remuneração do respondente, de forma a verificar se há diferenças de percepções nos alunos que transferiram seu turno de estudo durante o curso, bem como se níveis de remuneração mais altos têm efeito positivo na crença dos estudantes sobre sua carreira e seu desempenho acadêmico.

Isso significa que $\mathrm{o}$ ensino superior tem um papel importante no desenvolvimento da autoeficácia dos alunos, o que pode colocar a IES em um papel mais ativo na crença das capacidades que seus estudantes possuem. Dessa forma, evidencia-se a importância da promoção de ações que visem desenvolver as 
crenças de autoeficácia, tendo em vista que as percepções dos indivíduos têm consequências reais sobre as situações e seus comportamentos (NAYLOR; PRITCHARD; ILGEN, 1980).

Neste estudo não foi proposta a discussão de metodologias para o desenvolvimento da autoeficácia, o que requereria novas e detalhadas pesquisas, todavia não são recentes os estudos que destacam o impacto da interação entre indivíduo e ambiente no desenvolvimento da autoeficácia percebida (BANDURA; CERVONE, 1983), de forma que crenças e comportamentos podem ser modificados positivamente por meio de atividades estimulantes, que conduzam os universitários a gradativamente se perceberem como capazes de superar e vencer os desafios típicos do ensino superior.

A partir dessa perspectiva, finaliza-se ressaltando a responsabilidade das IES na criação de práticas que favoreçam nos seus estudantes crenças de autoeficácia mais robustas, subsidiando-os para enfrentar os desafios que se apresentarão no decorrer do desenvolvimento da sua carreira.

\section{ANA LÚCIA PEREIRA}

Doutoranda em Educação pela PUC-SP. Mestre em Educação. Graduada em Psicologia pela Universidade São Judas Tadeu.

TATIANI DOS SANTOS ZUPPANI

Doutoranda em Administração pela USP. Mestre em Administração pela Universidade Federal do Paraná, Graduada em Psicologia pela Universidade Federal de São Carlos.

MARCELO NEVES GONÇALVES

Doutorando em Administração pela USP. Mestre em Administração pela Universidade Presbiteriana Mackenzie, Bacharel em Administração de Empresas pela USP.

\section{Referências}

BANDURA, A.; AZZI, R. G.; POLYDORO, S. Teoria Social Cognitiva: conceitos básicos. Porto Alegre: Artmed, 2008.

BANDURA, A. Self-efficacy: the exercise of control. New York: W.H. Freeman, 1997.

BANDURA, A. Selff-efficacy in Change Societies. New York: Cambridge University Press, 1995. 
BANDURA, A. Exercise of personal agency thought the self-efficay mechanism. In: Schwarzer (Org.) Selff-efficacy: thought control of action Washington. Hemisphere: 1992, p. 3-38.

BANDURA, A. Pensamiento y Acción: fundamentos sociales. Barcelona: Martinez Rocca, 1987.

BANDURA, A.; CERVONE, D. Self-evaluative and self-efficacy mechanisms governing the motivational effects of goal systems. Journal of Personality and Social Psychology, n. 5, p. 1017-1028, 1983.

CORRAR, L. J.; PAULO, E.; DIAS FILHO, J. M. Análise Multivariada para os cursos de Administração, Ciências Contábeis e Economia. São Paulo: Editora Atlas, 2009.

FÁVERO, L. P.; BELFIORE, P.; DA SILVA, F. L.; CHAN, B. L. Análise de Dados: modelagem multivariada para tomada de decisões. Rio de janeiro: Campus, 2009.

FONTES, A. P.; AZZI, R. G. Crenças de autoeficácia e resiliência: apontamentos da literatura sociocognitiva. Estudos de Psicologia, Campinas, v. 1, jan./mar. 2012, p. 105-114.

HAIR JR., J.; ANDERSON, R. E.; TATHAM, R. L.; BLACK, W. C. Análise Multivariada de Dados. Porto Alegre: Bookman, 2005.

MINUSSI, L. A.; DAMACENA, C.; NESS JR., W. L. Um modelo de previsão de solvência utilizando regressão logística. Revista de Administração Contemporânea RAC, v. 6, n.3, set./dez., 2002.

NAYLOR, J.; PRITCHARD, R.; ILGEN, D. Organizational Climate and a Theory of Behavior in Organizations. New York: Academic Press, 1980.

SCHULTZ, D.; SCHULTZ, S. E. Teorias da personalidade. São Paulo: Cengage Learning, 2008. 